\title{
Real time prediction of track while scan data
}

\author{
Olga Selezneva ${ }^{1, a}$ \\ ${ }^{1}$ Saint Petersburg State University, Applied Mathematics Department, 198504 Saint-Petersburg, Russia
}

\begin{abstract}
The article covers several path prediction methods based on Kalman filter, compares them and estimates their advantages and disadvantages on example of searching a path of a maneuvering object on noisy pre-processed radar picture by evaluating their error values.
\end{abstract}

\section{Task description}

There is an ordered series of pictures (radar images), received at a fixed intervals of time. On one of them, we select a mark (tracking object). The task is to find this mark on the following images and to built its path of moving [1]. In is considered that the images are situated in the same coordinates (the radar is stationary), and the number of moving marks on the images is limited (sea vessels).

On the figure below there is a path built upon the center points of found marks. As in can be seen on the picture, it results rough because of mark positioning errors due to noisiness of images.

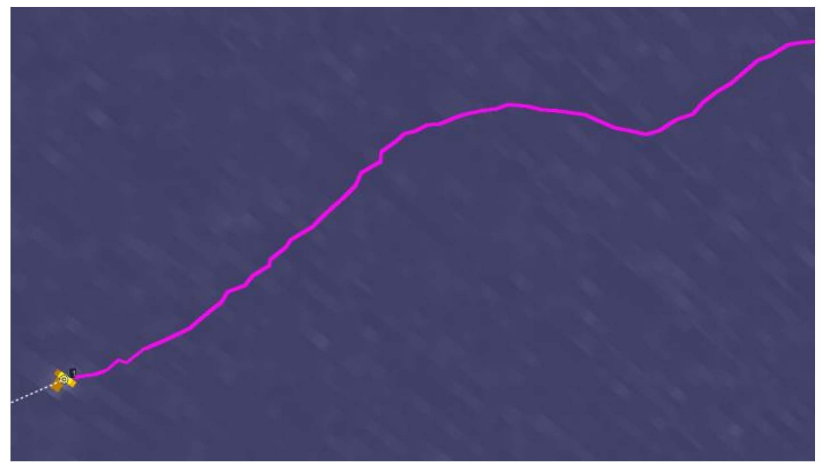

Figure 1. An example of a path, built based on noisy data.

The parameters of error in the geometric positions of objects are also unknown: they can appear just as because of the inaccuracy of the equipment (improper calibration of radar, imprecision of the equipment, noise on the data transmitting line), so due to the weather conditions, general situation and primary processing of images (for example, noise elimination and color correction) for finding geometric parameters of the mark. That is why for the further studying we will have to use simplified models of moving [2-4] and introduce a number of assumptions.

\section{Kalman filter}

Let us study a system:

$$
\begin{gathered}
x_{k}=F x_{k-1}+G a_{k} \\
z_{k}=H x_{k}+\vartheta_{k}
\end{gathered}
$$

Here $x_{k}$ is a vector of projections of coordinates and velocities of the object to the axes $\mathrm{Ox}$ and $\mathrm{Oy}$ :

$$
x_{k}=\left[\begin{array}{c}
x \\
\dot{x} \\
y \\
\dot{y}
\end{array}\right] ;
$$

According to the Newtonian mechanics, we can write:

$$
\begin{gathered}
F=\left[\begin{array}{cccc}
1 & \Delta t & 0 & 0 \\
0 & 1 & 0 & 0 \\
0 & 0 & 1 & \Delta t \\
0 & 0 & 0 & 1
\end{array}\right] ; \\
G=\left[\begin{array}{cccc}
\Delta t^{2} / 2 & 0 & 0 & 0 \\
0 & \Delta t & 0 & 0 \\
0 & 0 & \Delta t^{2} / 2 & 0 \\
0 & 0 & 0 & \Delta t
\end{array}\right] ; \\
a_{k}=\left[\begin{array}{l}
a_{x} \\
a_{x} \\
a_{y} \\
a_{y}
\end{array}\right] ;
\end{gathered}
$$

$a_{k}-$ is an unknown vector of acceleration projections. We will consider that between revolutions $(\mathrm{k}-1)$ and $\mathrm{k}$ the object is moving with steady acceleration, which is limited by the capacities of sea vessels.

Covariance matrix of random interaction is:

$$
Q=E\left[G a_{k}\left(G a_{k}\right)^{T}\right]=G\left[a_{k} a_{k}^{T}\right] G^{T}
$$

\footnotetext{
a Corresponding author: olga.v.selezneva@mail.ru
} 
$z_{k}-$ is a vector of measurements of object's coordinates for every revolution.

$$
H=\left[\begin{array}{llll}
1 & 0 & 1 & 0
\end{array}\right]
$$

$\vartheta_{k}$ - inaccuracy of measurements. Due to the fact that the real value of inaccuracy is unknown, we will consider it to have normal distribution with zero mathematical expectation and mean square deviation $\sigma_{z}$. In this, the measurement noise covariance matrix will look like:

$$
R=\left[\sigma_{z}^{2}\right]
$$

As the noise on the estimated images is monotonous, the adjustment of noise matrix $\mathrm{R}$ is to be performed only once.

Further, on the each step the stages of extrapolation and correction are used. The result of this work is shown below.

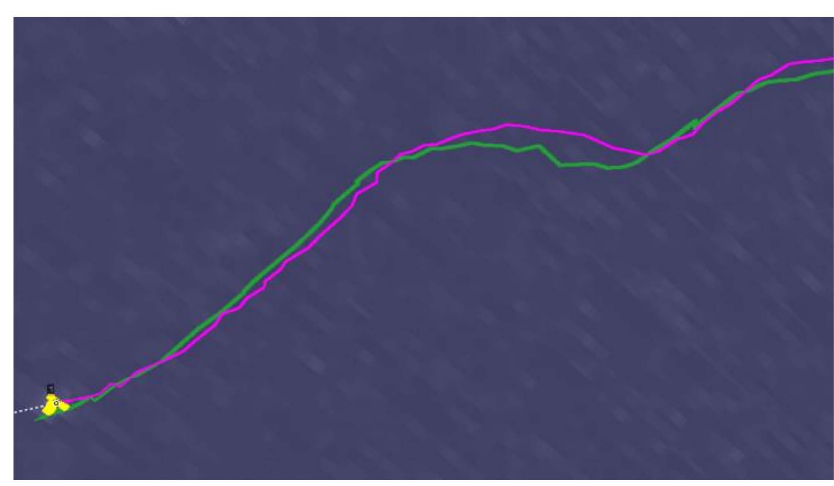

Figure 2. Comparison of a path constructed by Kalman's method and a path built on the centers of marks.

The Figure 2 shows two paths. The violet one is the path shown before, which is built upon the centers of found marks. The green one is a path, connecting the points received out of the presented model, which describe a predictable situation of the object. As it is seen from the figure, the received path goes closely enough to the one built upon the entering data. However, at the moment of maneuver, the paths are slightly separating.

Table 1. Values of errors for different objects

\begin{tabular}{|c|c|c|c|c|}
\hline Object & $\mathrm{N}=30$ & $\mathrm{~N}=40$ & $\mathrm{~N}=50$ & $\mathrm{~N}=60$ \\
\hline 1 & 6864.07 & 7257.87 & 8129.73 & 8438.21 \\
\hline 2 & 6850.12 & 9109.79 & 12022.4 & 14179.8 \\
\hline 3 & 21391.6 & 23961.2 & 27354.9 & 28560.4 \\
\hline 4 & 7684.74 & 11508.8 & 14656.9 & 17017.6 \\
\hline
\end{tabular}

The Table 1 represents the values of errors calculated according to the following formula:

$$
\operatorname{Err}(\mathrm{N})=\sum_{\mathrm{i}=1}^{\mathrm{N}} \sum_{\mathrm{j}=1}^{\mathrm{n}}\left(x_{i, j}^{o p t}-z_{i, j}\right)^{2}
$$

Here $x_{i, j}^{o p t}$ are the values of coordinates of the object calculated using the filter for $\mathrm{N}$ tracking points, $z_{i, j}-$ values of measurements, $\mathrm{n}=2$ is space dimension of calculation.
The values in the table are calculated for four different tracks and for different number of measurements.

\section{Conclusion}

The negative side of this method for the task is the absence of data about errors of the model and the measurements, we have to use average empiric values, and this causes unpredictable errors. What is more, for different objects the optimal value will differ. Another disadvantage is a complexity of programming and resource costs for calculation. However, with enough computation capacities, this method is able to provide a higher accuracy of the path

\section{References}

1. S.Z. Kuzmin, The basics of design of digital processing systems of radar data (1986)

2. R.E. Kalman, Journal of Basic Engineering, 82, 35 (1960)

3. P. Matisko, 16th IFAC Symposium on System Identification, pp. 1523 (2012)

4. W.A. Fuller, Measurement Error Models (1987) 